\title{
Loop Antenna Characterization for ELF and SLF Measurements
}

\author{
Caracterización de Antenas Lazo para Mediciones en ELF y SLF \\ G. I. Quintana*1, R. Alonso*2 and W. G. Fano*3 \\ ${ }^{\dagger}$ Universidad de Buenos Aires, Electronic Department, Electromagnetic Radiation Laboratory \\ Paseo Colon 850. (1063) Buenos Aires. Argentina \\ 1 gonza.quintana94@gmail.com \\ 2 ninjaramiro@gmail.com \\ ${ }^{3}$ gustavo.fanodieee.org \\ Recibido: 15/06/18; Aceptado: 26/07/18
}

\begin{abstract}
Electromagnetic Fields are present in the Earth, due both to natural and artificial emissions. The Electric and Magnetic fields generated by natural events like volcanic eruptions and earthquakes, are aspects of tectonomagnetism, volcanomagnetism and tectonoelectricity, in the electromagnetic spectrum from radio frequencies (RF) to submicrohertz frequencies. This paper is dedicated to the characterization of loop antennas for measuring electromagnetic precursors of seismic movements and other ELF (3-30 Hz) and SLF (30-300 $\mathrm{Hz}$ ) natural effects. The antenna factor, impedance, quality factor $Q$ and sensitivity of the antennas are measured. These characteristics are of great importance in the choice of the loop antenna to be used in the framework of the UBACyT research proyect Study of Electromagnetic Disturbances Produced by Seismic Movements.
\end{abstract}

Keywords: Electromagnetic Precursors, Magnetic Field, Loop Antenna, Antenna Factor.

Resumen- Los campos electromagnéticos que se encuentran presentes en la Tierra, son debidos tanto a emisiones de la naturaleza como producidos por el ser humano. Los campos eléctricos y magnéticos que son generados por eventos naturales como erupciones volcánicas y terremotos, son aspectos de tectomagnetismo, volcanomagnetismo y tectoelectricidad, en el espectro electromagnetico desde las frecuencias de radio (RF) hasta las frecuencias de los submicrohertz. Este trabajo se dedica a la caracterización de antenas lazo para medir los precursores electromagnéticos de movimientos sísmicos junto con otros efectos naturales en ELF (3-30 Hz) y SLF (30-300 Hz). El factor de antena, la impedancia, el factor de calidad $Q$ y la sensibilidad de las antenas son medidos. Estas características son de vital importancia para la elección de la antena lazo a utilizar en el marco del proyecto de investigación de UBACyT Estudio de Perturbaciones Electromagnéticas Producidas por Movimientos Sísmicos.

Palabras clave: Precursores Electromagnéticos, Campo Magnético, Antena lazo, Factor de Antena.

\section{INTRODUCTION}

During the past few decades, a remarkable increase in the quality and quantity of electromagnetic data recorded before and during eruptions and earthquakes [1] are evidence that seismic movements are preceded by anomalous electromagnetic signals.

The Electromagnetic Precursors signals have been discussed in many publications [1], [2], [3] and [4]. The most accepted theory is that electromagnetic waves are generated as a consequence of microfractures in the rocks. The electric charges of opposite sign, created on opposite sides of the microfractures form electric dipoles separated by a distance $d$. This separation is modulated by the mechanical vibrations of its walls (originated by rupture of the atomic bonds), giving rise to dipole oscillations and electromagnetic waves [5]. This emissions are in the ELF $(3-30 \mathrm{~Hz})$ and TLF $(<$ $3 \mathrm{~Hz}$ ) range.

Another natural phenomena at ELF are the Schumann resonances, which are electromagnetic resonances in the cavity formed by the ionosphere and Earth's surface (that can be modeled as a conductor for low frequencies) excited by lighting discharges. The first 3 peak frequencies, $7.8 \mathrm{~Hz}$, $14 \mathrm{~Hz}$ and $20 \mathrm{~Hz}$, had been measured at the location of Villa Alpina, Córdoba, Argentina [6]. This measurement had the purpose to get evidence of electromagnetic precursors of earthquakes in a quiet zone. This location was chosen due to the very low artificial noise of the power lines radiation [6].

The most important naturally occurring VLF signal is the whistler. A whistler is created from a lightning stroke that passes first to the ionosphere and then to the magnetosphere above. These particles are then guided along the Earth's magnetic field, returning to ground to the opposite hemisphere [2].

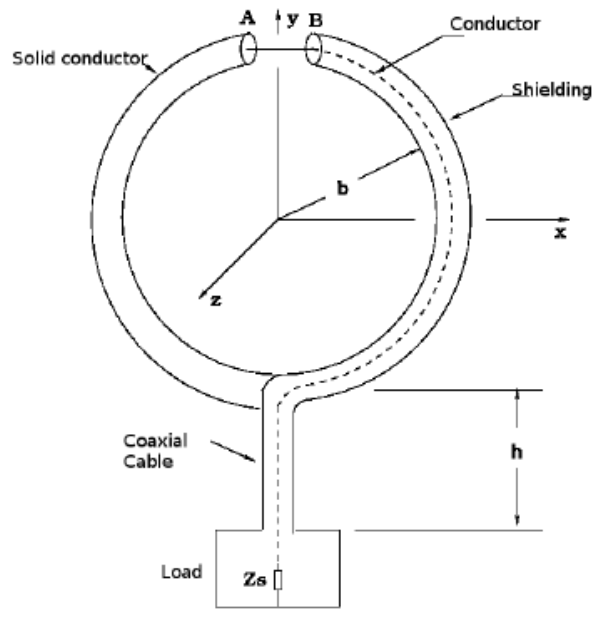

Fig. 1. Shielded loop antenna. 


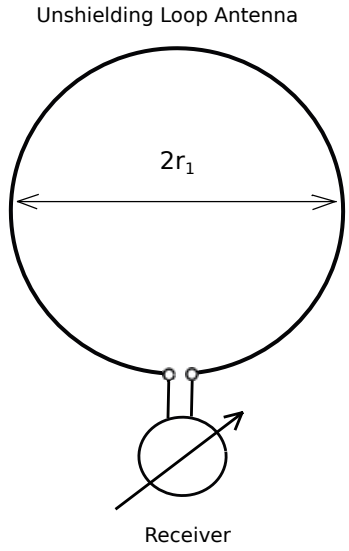

Fig. 2. Unshielded loop antenna.

Due to the Electromagnetic Compatibility regulations (EMC), measurements of Electric and Magnetic Fields are of great importance. A very useful method for measuring the Magnetic Field of electromagnetic waves is by means of a loop antenna, which can be shielded or unshielded. A shielded loop antenna can be seen in Figure 1 and an unshielded loop in Figure 2. The radiation pattern of a loop antenna, in which the size of the loop is much smaller than the wavelength, is described in Figure 3. As all measurements are made within the ELF and SLF ranges, Figure 3 represents the directional dependence of the received power of the antennas used in this work.

The unshielded loop will receive the electromagnetic radiation and the quasistatic induced noise. This noise will typically come from fluorescent lamps, brush motors, corona discharge from HV power lines, and the radiation from the line scan of TV sets [7]. The shielding loop, on the other hand, is practically insensitive to stray fields induced noise, which is quite important as nearly all of the energy content of long wave and medium wave interference locally generated is quasistatic [7]. The gap observed in Figure 1 in the shielding, prevents the currents around the loop due to possible atmospheric effects and electrostatic discharges [8]. Magnetic loops have long been used by EMC personnel to "sniff" out sources of emissions in circuits and equipment [8].

In order to measure the ELF and SLF spectrum, two circular loop antennas are constructed and compared. These loops, together with a low noise amplifier and a computer (using the free software GNU-Radio to compute the FFT), allow to sense the normal component of the incident Magnetic Field and it's frequency spectrum. By using an array of three of this loops, the 3 components of $\vec{B}$ can be measured.

This paper is dedicated to the characterization and comparison of these two antennas. As all the measurements have been made in the laboratory, in the absence of those undesired effects, unshielded loops are used. In the future, when using these loops for capturing electromagnetic precursors and other natural electromagnetic emissions (as Schumann resonances), a suitable shield will be needed to avoid the above mentioned problems.

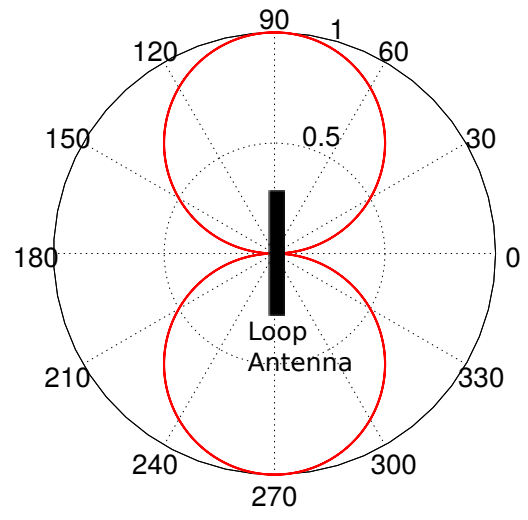

Fig. 3. Radiation patterns of a circular short loop antenna.

\section{ANTENNA FACTOR}

The Antenna Factor or Correction Factor of a loop antenna relates the magnitude of the incident Magnetic Field (H) and the induced voltage (open circuit voltage) at the antenna terminals $(\mathrm{V})$. It is defined thus:

$$
K=\frac{H}{V} \quad\left[\frac{A}{V m}\right]
$$

The Maxwell-Faraday Law expression in differential form is [9]:

$$
\vec{\nabla} \times \vec{E}=-\frac{\partial \vec{B}}{\partial t}
$$

where:

$B[T]$ : is the magnetic flow density.

$E[V / m]:$ is the electric field.

$t[s]:$ is the time.

In order to obtain the open circuit induced voltage at the terminals of the loop antenna, integration is performed at both sides of eqn. (2) :

$$
V_{o c}=-\frac{\partial \phi}{\partial t}
$$

where: $\phi=\int \vec{B} \cdot \overrightarrow{d s}$ is the magnetic flux.

Consider a short single turn loop, excited by a harmonic electromagnetic wave as a function of time where the wavelength of the electromagnetic wave received is much greater than the perimeter of this loop. The magnetic flux is then:

$$
\phi=B \int d s=\mu_{0} H A
$$

The open circuit induced voltage of $\mathrm{N}$ turns of the loop antenna is [9]:

$$
V_{o c}=N A \mu_{0} \frac{\partial H}{\partial t}
$$

This voltage can be written thus [10].

$$
V_{o c}=N \omega \mu_{0} H \cos \theta A
$$

where $\theta$ is the angle between $\mathrm{z}$ and $\mathrm{H}$ and $\omega=2 \pi f$. 
Using eqn. (6) and admitting that the incident magnetic field is parallel to the antenna's axis, the antenna factor is obtained:

$$
K=\frac{1}{N \omega \mu_{0} A}
$$

which in $\mathrm{dB}$ is a straight line with a slope of $-20 \mathrm{~dB} / \mathrm{decade}$. As in this case the loop antenna has no ferromagnetic core, we have $\mu_{r}=1$.

The short loop antenna is usually a very inefficient antenna [11], which can be observed by means of its Radiation resistance in transmitting mode. However, this is not of importance in the receiving mode, where the knowing the antenna factor with precision takes real importance.

\section{SENSITIVITY}

The Sensitivity of a loop antenna states the minimum Magnetic Field that can be measured. Thermal noise can be expressed thus [12]:

$$
E_{a}=(4 k T R a)^{1 / 2}\left[V \cdot H z^{-1 / 2}\right]
$$

where $k$ is the Boltzmann constant, $T$ the temperature in Kelvin and $R a$ the antenna's resistance. The Voltage induced by a uniform Magnetic Field in a loop antenna of area $A$ and $N$ turns is, from Faraday's law

$$
V_{o c}=\omega N A B \quad[V]
$$

Combining these two equations, the equivalent magnetic field of the antenna's thermal noise is:

$$
B=\frac{\sqrt{4 k T R}}{N A \omega}\left[T \cdot H z^{-1 / 2}\right]
$$

Normalizing the last result by the factor $1 / f$, the sensitivity is [12], [13]:

$$
S_{a}=\frac{\sqrt{4 k T R}}{2 \pi N A} \quad\left[T \cdot H z^{1 / 2}\right]
$$

The sensitivity is simply the magnetic field equivalent value of the antenna's thermal noise in a $1 \mathrm{~Hz}$ of bandwidth, normalized by the factor $1 / f$ [12]. Consequently, it gives the minimum Magnetic Field that can be measured (Magnetic Fields of less magnitude would be hidden by thermal noise).

\section{Results}

In order to measure Magnetic Fields at low frequencies, two circular short loop antennas have been designed and constructed (see Figure 4). For measuring the Electric Field of the electromagnetic wave instead, short Dipole Antennas are frequently used [14].

The characteristics of these two loops antennas, of $N=$ 180 and $N=300$ turns each, are listed in Table I. In that table, $r_{c}$ is the conductor radius, $r_{l}$ the loop radius, $\mu_{r}$ and $\epsilon_{r}$ the relative permeability and permitivity, $f_{0}$ the resonance frequency and $R_{D C}$ the antenna's series resistance (at low frequencies).

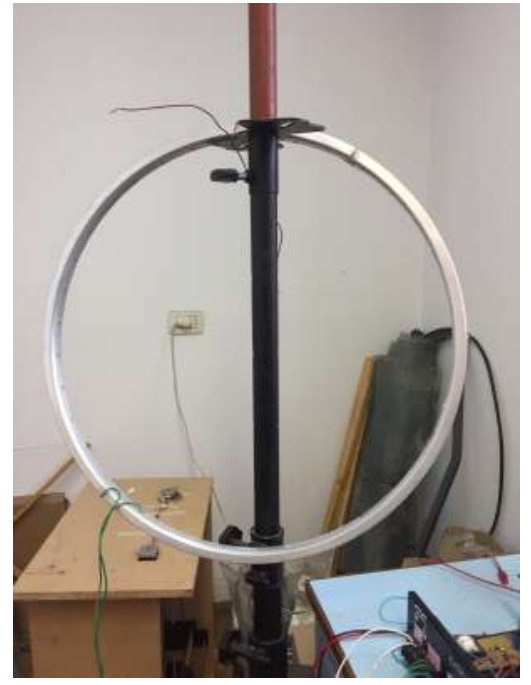

Fig. 4. One of the loop antennas constructed.

TABLE I

LOOP ANTENNA CHARACTERISTICS

\begin{tabular}{|l||l||l|}
\hline$N$ & 180 & 300 \\
\hline$\epsilon_{r}$ & 4 & 4 \\
\hline$\mu_{r}$ & 1 & 1 \\
\hline$r_{l}(m)$ & 0.28 & 0.28 \\
\hline$r_{c}(m m)$ & 0.35 & 0.35 \\
\hline$f_{0}(k H z)$ & 24 & 17 \\
\hline$L(m H)$ & 64 & 120 \\
\hline$R_{D C}(\Omega)$ & 14.5 & 23.7 \\
\hline
\end{tabular}

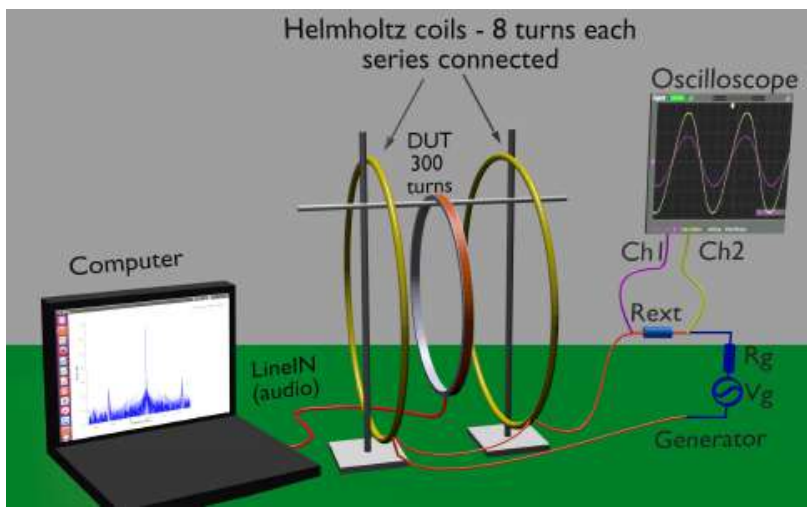

Fig. 5. Antenna Factor Measurement setup.

\section{A. Antenna Factor Measurement}

Using the Helmholtz coils [15] and the measurement scheme in Figure 5, a uniform Magnetic Field is generated in the space between the coils, in which the loop antenna is placed.

The magnitude of this Field depends on the geometrical characteristics of the Helmholtz coils and the current flowing trough them. This Magnetic Field can be computed with equation [15]:

$$
H=\frac{0.7154 N_{h} I}{r_{h}}
$$

where $N_{h}=8$ is the number of turns of each Helmholtz coil and $r_{h}=39 \mathrm{~cm}$ is their radius. By setting $I=20 \mathrm{~mA}$, the Magnetic Field is fixed in $H=0.2057 \mathrm{~A} / \mathrm{m}$. This Magnetic Field induces a voltage in the antenna under test, which 
is captured by the data acquisition system of a personal computer with the software GNU-Radio (which has been previously calibrated) and showed in a scope. In order to assure a constant current $I$, a test resistor $R_{p}=56 \Omega$ is introduced and its voltage drop is continuously monitored. As $I=20 \mathrm{~mA}$, this peak voltage drop should always be $V_{p}=1.12 \mathrm{~V}$.

The frequency of this current, and therefore of the Magnetic Field, is swept from $5 \mathrm{~Hz}$ to $10 \mathrm{kHz}$ and the different amplitudes captured in the scope of the software GNU-Radio are noted.

As the Magnetic Field $\mathrm{H}$ is fixed, using eqn. (1), the frequency response of the Antenna Factor of the two antennas is computed. This measurement and the theoretical curves are compared in the Figures 6 and 7.

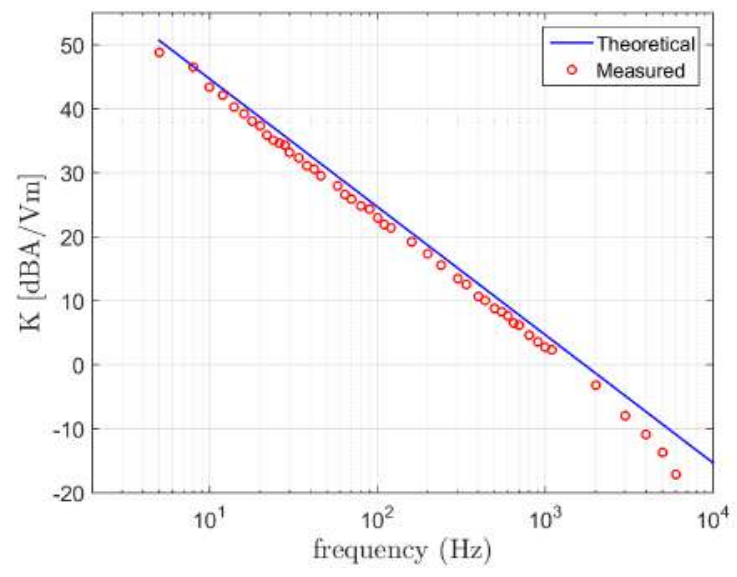

Fig. 6. Antenna Factor of the loop with 300 turns.

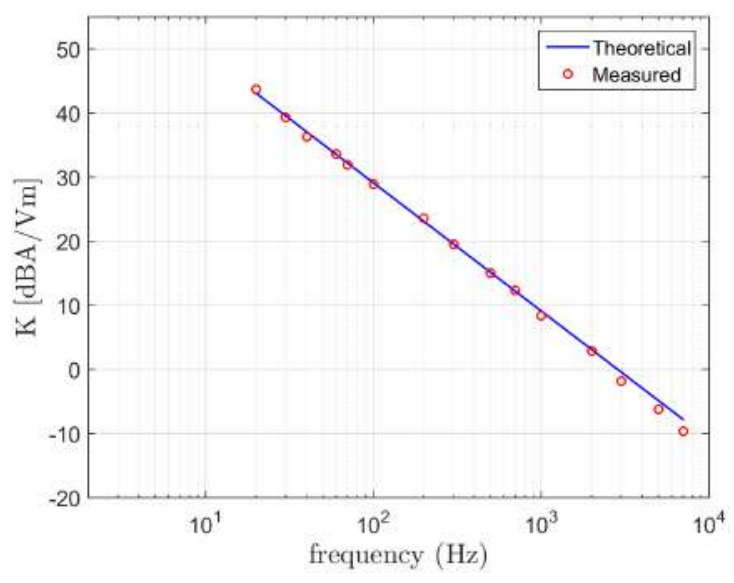

Fig. 7. Antenna Factor with the loop of 180 turns.

It can be seen from Figure 6 that the measured Antenna Factor for $N=300$ turns follows well the theoretical curve for frequencies of up to $2 \mathrm{kHz}$. As we move towards the system's resonance frequency, the rate of increase of the reactance of the coils is increased, which triggers the induced voltage. As the Magnetic Field $\mathrm{H}$ continues being constant this results in a decrease of the Antenna Factor, as can be seen in Figure 6.

In contrast, the above mentioned effect can't be seen in
Figure 7 as the resonance frequency of this loop antenna is higher than of the larger one.

\section{B. Antenna Impedance Measurements}

The frequency response of the antenna impedance, both the resistance and the reactance, for the two loops are measured with a Gwinstek LCR-819 LCR-meter. These results are shown in Figure 8 y 9.

It can be seen that the antennas behave as inductors in the range of frequencies of interest, as their reactance augments linearly with frequency. However, the resistance rises for frequencies near $500 \mathrm{~Hz}$. To explain this phenomena, the behavior of the impedance's resistive part of a long wire, which is detailed in [16], is examined. The general expression for the interior impedance of a long straight wire is:

$$
Z_{\text {wire }}=\frac{L \sqrt{j \omega \mu /(\sigma+j \omega \epsilon)}}{2 \pi a} \frac{I_{0}(\gamma a)}{I_{1}(\gamma a)}
$$

where $\gamma \approx \sqrt{j \omega \mu \sigma}$ is the propagation constant, $I_{0}$ and $I_{1}$ are the modified Bessel functions, $a$ is the wire's radius and $L$ is its length.

At low frequencies, where the wire radius is small compared with the wave's depth of penetration, the expression of eqn. (13) is reduced to [16]:

$$
Z_{\text {wire }} \approx \frac{L}{\pi a^{2} \sigma}+j \omega \frac{\mu L}{8 \pi}
$$

This equation can be used to estimate theoretically the loops resistances at low frequencies. The antennas under test were constructed with a copper cable of diameter 0.7 $\mathrm{mm}$. Considering $\mu=\mu_{0}$ and $\sigma_{C u}=5.9610^{7} \mathrm{~S} / \mathrm{m}$, the impedance of two long wires (of the same length that the loops) at low frequencies are then

$$
\begin{gathered}
Z_{N=180} \approx 13.8 \Omega+j \omega 15.84 \mu H \\
Z_{N=300} \approx 23 \Omega+j \omega 26.4 \mu H
\end{gathered}
$$

It can be seen that the resistances obtained with equation 13 are practically equal to the measured $14,5 \Omega$ and $23,7 \Omega$ at low frequencies. In addition, the contribution of the internal impedance to the total inductance of the loop is negligible for low frequencies.

At higher frequencies the depth of penetration gets smaller, which causes the wire's resistance to increase as the conducting area is reduced (this is known as Skin Effect). When the depth of penetration is small compared to the radius of the wire, eqn. (13) is reduced to

$$
Z_{\text {wire }} \approx \frac{1}{2 \pi a} \sqrt{\frac{\omega \mu}{2 \sigma}}+\frac{j}{2 \pi a} \sqrt{\frac{\omega \mu}{2 \sigma}}
$$

For a copper wire of $0.7 \mathrm{~mm}$ of diameter the increase of the resistance starts being detected at frequencies from 10 to $100 \mathrm{kHz}$, which are much higher frequencies than the 500 $\mathrm{Hz}$ at which this effect is seen in Figure 8. However, eqn. (17) stands for a straight long wire, which is not our case. In coiled-wire inductors, Skin Effect is enhanced by the Proximity Effect. The magnetic flux through a conductor turn generated by the current in an adjacent turn produces a circulating current or Eddy current in the conductor which 
results in an increase of the wire's resistance [17].

Additionally, a radial resistivity gradient (high resistivity at the outer side and low resistivity at the inner one) develops in the bent wire. This is consequence of both the difference in conduction paths between wire ends and the rearrange of the microcrystals at the material's bulk due to stretching [17].

This two effects drag the increase of the antenna's resistance to lower frequencies, therefore providing a theoretical explanation of Figure 8 .

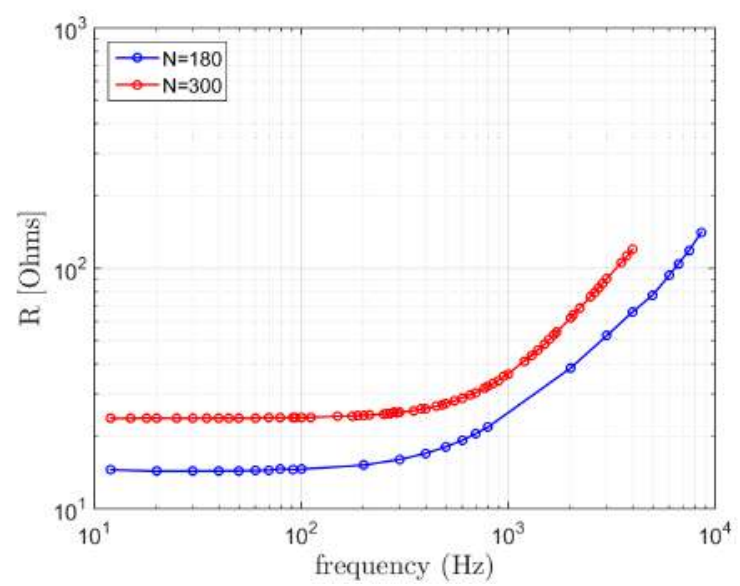

Fig. 8. Resistance of the two Loop Antennas.

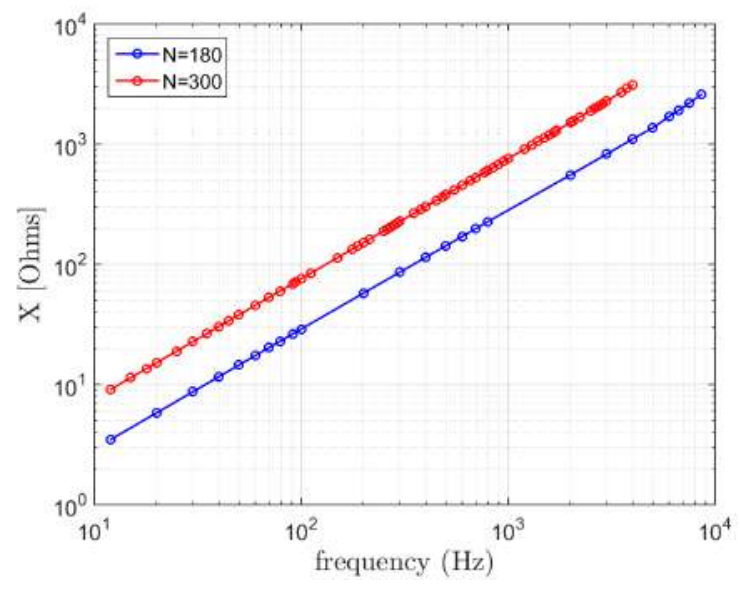

Fig. 9. Reactance of the two Loop Antennas.

The Quality Factor, which is defined as $\frac{\omega L}{R}[18]$ is plotted in Figure 10. The $\mathrm{Q}$ increases with frequency up to 20 (for $\mathrm{N}=180$ ) or 30 (for $\mathrm{N}=300$ ), due to the increase of the resistance of the loop antennas with frequency.

1)

\section{Sensitivity of the Antennas}

The Sensitivity of three different antennas, the two previous circular loops and one rectangular loop antenna, which was used in [6] to measure the Schumann Resonance, have been computed using eqn. (11). The results are listed in Table II, being $R a$ the series antenna resistance, $A$ the loop area, $r_{c}$ the conductor radius and $S a$ the sensitivity.

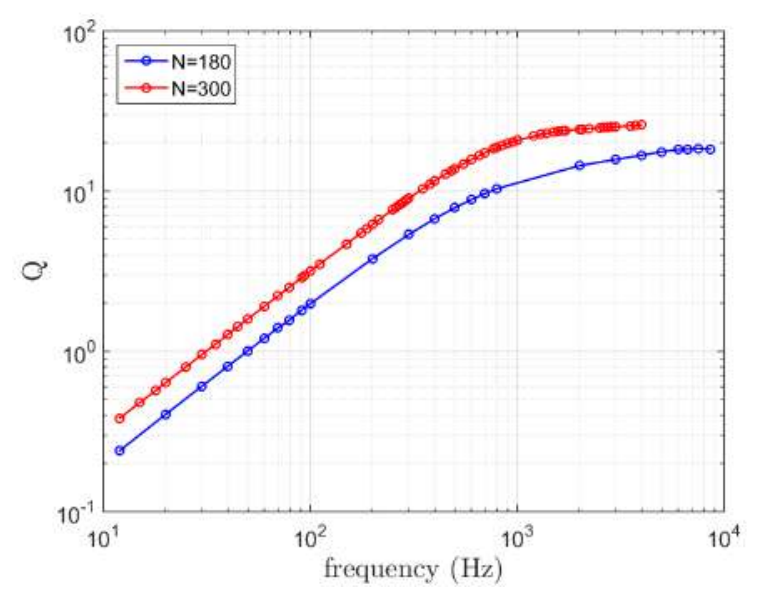

Fig. 10. Quality Factor Q as a function frequency.

TABLE II

SENSITIVITY OF THE THREE LOOP ANTENNAS

\begin{tabular}{|c||c||c||c||c|}
\hline$N$ & $R a[\Omega]$ & $A\left[m^{2}\right]$ & $r_{c}[m m]$ & $S a\left[p T H z^{1 / 2}\right]$ \\
\hline 180 & 14.5 & 0.2463 & 0.35 & 1.68 \\
\hline 300 & 23.75 & 0.2463 & 0.35 & 1.28 \\
\hline 100000 & $16 k$ & $64 \cdot 10^{-4}$ & 0.12 & 3.86 \\
\hline
\end{tabular}

The sensitivity values of the loop antennas, as these are going to be used to measure seismic electromagnetic precursors and Schumann resonances, should enable them to capture Magnetic Fields of tens of pico Tesla.

Considering a bandwidth of $1 \mathrm{~Hz}$, the minimum Magnetic Field that can be measured with the 180 and 300 turns loops is $1.68 \mathrm{pT}$ and $1.28 \mathrm{pT}$, respectively. Hence, the two constructed loops are capable of measuring these natural effects, being the larger loop fitter than the smaller, as its sensitivity is lower. Moreover, as these two have a lower sensitivity than the loop used in [6], they should also be used to measure Schumann resonances (in conjunction with a proper high gain and low noise amplifier).

\section{CONCLUSIONS}

Two circular loop antennas, one with 180 and the other with 300 turns, have been constructed and characterized, in order to test their fitness for measuring low amplitude magnetic fields at ELF. As the only difference between them is the number of turns, the one with more turns has a bigger inductance (providing though a higher amplification of the signal under test) but has a larger series-resistance in return. In order to make precise measurements, the loops have been calibrated using a pair of Helmholtz coils [15] and their antenna factor have been measured for frequencies up to $10 \mathrm{kHz}$. These measurements are in agreement with the theoretical Antenna Factor curves.

Additionally, the antenna impedance of the two loops have been measured in a wide frequency range (from ELF to VLF). It is concluded that the loops can be used as measuring devices for frequencies lower than $1 \mathrm{kHz}$. For higher frequencies, as a consequence of the skin effect enhanced by the proximity effect, the antenna resistance grows and the Q-factor stagnates. This, added to the fact that the loop approaches its resonance, makes the antenna to 
stop behaving as a an inductor, making it though unsuitable for measuring magnetic fields.

Finally, the sensitivity of the two loop antennas have been computed and compared to the sensitivity of the antenna used in [6] and to the expected amplitude of the magnetic seismic precursors. As the minimum Magnetic Field that can be captured with each loop is lower than those naturally induced fields, it is then concluded that the coils are fit to be used in the UBACyT project Study of Electromagnetic Disturbances Produced by Seismic Movements, which is being carried out by the Electromagnetic Radiation Laboratory, in conjunction with other laboratories of this University. As the 300 turns coils has been showed to have lower sensitivity than the one with 180 turns and as it provides a higher signal amplification, the authors conclude that the larger loop is preferable to the smaller one.

\section{ACKNOWLEDGMENT}

The authors would like to thank to the University of Buenos Aires and to the UBACyT research grants.

\section{REFERENCES}

[1] M. J. S. Johnston, "Review of electric and magnetic fields accompanying seismic and volcanic activity," Surveys in Geophysics, vol. 18, no. 5, p. 441-475, 1997.

[2] W. G. Fano, G. Quintana, and R. Alonso, "3d magnetic field measurement at elf and slf frequencies," IEEE 2018 GEMCCON (sent), 2018.

[3] A. C. F. Smith, A. Bernardi, P. R. McGill, M. Ladd, R. Helliwell, and O. Villard, "Low frequency magnetic field measurements near the epicenter of the ms loma prieta earthquake," Geophysical Research Letters, vol. 17, no. 9, pp. 1465-1468, August 1990.

[4] M. Parrot, J. Achache, J. Berthelier, E. Blanc, A. Deschamps, F. Lefeuvre, M. Menvielle, J. Plantet, P. Tarits, and J. P. Villain, "High frequency seismo-electromagnetic effects," Physics of the Earth and Planetary Interiors, pp. 65-83, 1993.
[5] E. R. Mognaschi, "On the possible origin, propagation and detectebility of electromagnetic precursors of eaerthquakes." Atti Ticinensi di Scienze della Terra., vol. 43, p. 111-118, 2002.

[6] E. Maffia, V. Trainotti, W. G. Fano, and N. Trench, "Medicion de la resonancia de schumann," Latinmag Letter, vol. 1, no. A02, pp. 1-8, 2011.

[7] D. C. Smith, "Current probes, more useful than you think," Electromagnetic Compatibility, 1998. 1998 IEEE International Symposium on, vol. 1, no. 2, pp. 284-289, 1998.

[8] D. Smith, "Signal and noise measurement techniques using magnetic field probes," Electromagnetic Compatibility, 1998. 1998 IEEE International Symposium on, no. 2, pp. 559-563, 1999.

[9] V. Trainotti and W. G. Fano, Ingenieria Electromagnetica, 1st ed., N. Libreria, Ed. Nueva Libreria, 2004, vol. 1.

[10] W. G. Fano, Electric Field. Chapter:Standard Electric and Magnetic Field for Calibration, M. Sheikholeslami, Ed. Intechopen, 2018. [Online]. Available: www.intechopen.com

[11] C. A. Balanis, Antenna Theory: Analysis and Design. WileyInterscience, 2005.

[12] M. B. Cohen, S. I. Umran, and E. W. Paschal, "Sensitive broadband elf/vlf radio reception with the awesome instrument," IEEE Transactions on Geoscience and Remote Sensing,, vol. 48, no. 1, p. 3-17, 2010.

[13] L. Minh Tan and K. Ghanbari, "Development of the new elf/vlf receiver for detecting the sudden ionospheric disturbances," Acto Technica Napocensis Electronics and Telecommunications, vol. 57, no. 1, p. 1-5, 2016.

[14] H. Trzaska, Electromagnetic Field Measurements in the Near Field. Noble Publishing Corp., 2001.

[15] W. G. Fano, R. Alonso, and G. Quintana, "El campo magnético generado por las bobinas de helmholtz y su aplicación a la calibración de sondas," Revista Elektron, vol. 1, no. 2, pp. 91-96, 2017.

[16] E. C. Jordan, Electromagnetics Waves and Radiating Systems. Prentice-Hall, 1950.

[17] H. Nagaoka, "The inductance coefficient of solenoids." Journal of College of Science, Imperial University, Tokio, Japan., vol. 27, no. 6,

[18] M. E. V. Valkenburg, Network Analysis. Prentice-Hall Incorporated, 1999. 